\title{
Analyse des déterminants et des conséquences des conflits locaux
}

Profils et stratégies des opposants et promoteurs de projets porcins

Profiles and strategies of projects' opponents and proponents in pig farming

Marie-Laurence Grannec, Mathilde Salinas, Yannick Ramonet, Philippe Boudes et Adel Selmi

\section{(2) OpenEdition Journals}

\section{Édition électronique}

URL : http://journals.openedition.org/economierurale/5120

DOI : 10.4000/economierurale.5120

ISSN : 2105-2581

Éditeur

Société Française d'Économie Rurale (SFER)

Édition imprimée

Date de publication : 17 février 2017

Pagination : 57-71

ISSN : 0013-0559

Référence électronique

Marie-Laurence Grannec, Mathilde Salinas, Yannick Ramonet, Philippe Boudes et Adel Selmi, « Analyse des déterminants et des conséquences des conflits locaux », Économie rurale [En ligne], 357-358 | janvier-mars 2017, mis en ligne le 17 février 2019, consulté le 21 décembre 2020. URL : http:// journals.openedition.org/economierurale/5120; DOI : https://doi.org/10.4000/economierurale.5120 


\title{
Analyse des déterminants et des conséquences des conflits locaux Profils et stratégies des opposants et promoteurs de projets porcins
}

\author{
Marie-Laurence GRANNEC, Mathilde SALINAS, Yannick RAMONET • Chambre régionale \\ d'agriculture de Bretagne, Rennes \\ marie-laurence.grannec@bretagne.chambagri.fr ; mathilde.salinas@bretagne.chambagri.fr ; \\ yannick.ramonet@bretagne.chambagri.fr \\ Philippe BOUDES • Agrocampus Ouest et UMR ESO CNRS, Rennes \\ philippe.boudes@agrocampus-ouest.fr \\ Adel SELMI • INRA UR 1326 SenS, Université Paris-Est, Champs-sur-Marne \\ Adel.Selmi@versailles.inra.fr
}

La production porcine est très concernée par la question de la représentation sociale de l'élevage, car elle symbolise souvent, pour le grand public, les inconvénients liés aux productions animales. De ce fait, les projets qui y sont liés sont plus fréquemment contestés. Synthétisant les principaux résultats d'une enquête menée entre 2012 et 2014 sur une quinzaine de cas de projets porcins conflictuels en Bretagne, cet article propose d'en analyser les déterminants. II explore les représentations de l'élevage et des éleveurs porcins, les registres d'argumentation et les stratégies adoptées par les acteurs opposés ou favorables aux projets. II propose des préconisations visant à favoriser la concertation territoriale et accompagner l'évolution des exploitations.

MOTS-CLÉS : élevage porcin, conflit local, négociation

\section{Profiles and strategies of projects' opponents and proponents in pig farming}

Among breeding activities, pig production is one of the most deeply involved in the question of social representation. Indeed, it often symbolizes for non-agricultural people the inconveniences related to livestock productions. So, pig farm extension projects are more often disputed. Summarizing the main results of two phases of inquiry (2012 and 2014), conducted on fifteen cases of conflicts related to porcine projects in Brittany, this report analyses determiners. Through the opinion of various actors in the territories, favorable or unfavorable to the projects, it compares their representations of pig producers and farms, their argument registers and their strategies. It allows the development of recommendations to favor territorial cooperation and to support the development of farms. (JEL: D74).

KEYWORDS: pig farming, local conflict, negotiation

D epuis plusieurs décennies, les éleveurs sont confrontés à un accroissement des interpellations sociales, liées notamment aux évolutions sociodémographiques du monde rural (Mathieu, 1998) et souvent appréhendées en termes de conflits d'usage et de voisinage (Torre et al., 2006). L'évolution des pratiques, l'agrandissement des élevages, leur concentration géographique ou le développement de systèmes hors sol ont contribué à l'émergence de controverses autour de la préservation de l'environnement puis, plus récemment, du mode d'élevage des animaux (Delanoue et al., 2014). La production porcine est plus particulièrement remise en cause car elle rassemblerait l'ensemble des inconvénients associés aux élevages, relativement 
aux conditions de vie des animaux; à l'impact environnemental, en particulier les nitrates et les algues vertes; et aux nuisances olfactives (Nicourt et al., 2000). Au niveau local, les conflits proviennent le plus souvent de la crainte ou de l'existence de nuisances et du risque de dépréciation immobilière. Les odeurs issues de l'élevage ou des épandages sont la principale source d'inquiétude (Nicourt et al., 2000) et l'un des premiers facteurs limitant le développement de ces élevages (Bonnaud et Nicourt, 2006). Les projets liés à ces élevages peuvent alors susciter un rejet et les arrêtés d'autorisation de porcheries sont davantage contestés que ceux des autres espèces puisque, à la mi 2011, $9 \%$ des arrêtés d'autorisation délivrés en 2010 pour des élevages porcins avaient été attaqués au tribunal administratif contre moins de $1 \%$ en production bovine ou avicole (Danel et al., 2012).

Cet article propose d'analyser cette apparente spécificité des conflits relatifs aux élevages porcins, en ciblant leur dimension locale. Sur l'ensemble du territoire breton, 16 cas de conflits ont été étudiés entre 2012 et 2014, avec la volonté de prendre en compte de manière symétrique les porteurs de projets et leurs opposants. En donnant la parole à d'autres acteurs que les éleveurs et leurs partenaires, ce travail apporte ainsi un éclairage nouveau et complémentaire de ceux déjà réalisés en production porcine, notamment par Nicourt et al. (2000).

Pour situer notre démarche, rappelons que les travaux sur les conflits démontrent de manière contre-intuitive leurs effets intégrateurs. C'est généralement l'analyse de Simmel ([1908] 1999; Duhaime, 2001) qui est mobilisée pour rappeler que le conflit, en tant qu'interaction entre individus ou groupes, participe de leur socialisation et doit être considéré comme un moment positif voire nécessaire. Les travaux plus récents, loin de contester cette vocation socialisatrice du conflit, insistent maintenant sur la nature des conflits, sur leurs enjeux et sur leurs dynamiques, et proposent des éléments de gestion ou de régulation de ces situations. La typologie des conflits d'aménagement de Dziedzicki (2003) illustre cette perspective : l'analyse des revendications des opposants dans le cadre de conflit d'aménagement permet d'identifier la nature de chaque conflit dans le but de « leur trouver une issue constructive ». Cette approche peut être rapprochée des travaux sur la démocratie participative, où la nature des mobilisations collectives se définit par rapport aux nouvelles formes de participation (e.g., Simard et al., 2006) ou par rapport à ce qui pousse les individus à entrer dans les débats et, par là, à donner vie au conflit (e.g., Barbier et Rémy, 2012).

Les thématiques des enjeux et des dynamiques sont liées à ces travaux sur la nature des conflits. Cela est particulièrement vrai dans le cas des espaces ruraux, qui ont vu les conflits - ou, plus certainement, les études sur le conflit - se multiplier, conséquence des évolutions des usages de ces espaces depuis les années 1970 et de ce qui est alors nommé « l'urbanisation des campagnes » (e.g., Mormont, 2006). La notion de conflits d'usage et de voisinage est alors reprise par la sociologie, la géographie ou l'économie. La synthèse de Torre et al. (2006) distingue alors trois fonctions concurrentielles de l'espace rural : productive, récréative et patrimoniale, en écho aux trois dimensions proposées auparavant par Perrier-Cornet (2002), relativement à une campagne ressource, paysage et nature.

Dans cet article, nous ciblons justement cette opposition entre une campagne ressource, dédiée aux usages agricoles, et ses autres dimensions, dans le but non seulement de contribuer à l'analyse des conflits et de leurs processus, mais également dans celui de les prévenir ou d'accompagner les parties prenantes dans une démarche la plus constructive possible - intégratice et 
socialisatrice pour reprendre les termes utilisés plus haut. Cet objectif, bien que lié ici à une collaboration à visée opérationnelle entre une Chambre d'agriculture et une Unité de recherche, apparaît par ailleurs dans la plupart des travaux sur le conflit. C'est ainsi que Thuderoz (2014) définit le conflit comme un événement régulateur, normal et utile, et ancre ses recherches dans le champ de la négociation. Étudier le conflit, dirait à nouveau Simmel ([1908] 1999), c'est étudier son «énergie unificatrice » et par là sa vocation intégratrice, comme les analyses sociales et spatiales de l'école de Chicago l'ont très tôt confirmé (e.g., Park, [1926] 1979).

En nous inscrivant dans cette dynamique, notre analyse des conflits relatifs aux projets de création ou d'agrandissement d'élevages porcins s'intéresse autant aux représentations ${ }^{1}$ que les protagonistes portent à l'égard des enjeux et des personnes impliquées, qu'aux dynamiques et aux conséquences du conflit pour les acteurs et les territoires engagés. Après une partie dédiée aux éléments de méthode, les cas d'étude sont abordés à partir des récits des personnes impliquées, de la distinction entre les porteurs de projet et les opposants, de l'analyse des représentations et des stratégies des parties prenantes. Enfin, bien qu'elle ne revienne pas directement sur les conséquences en termes de reterritorialisation de l'agriculture (Mormont, 2006 ; Darly, 2012), notre contribution propose des éléments de régulation et de prévention des conflits et rappelle, en conclusion, que les principales différenciations entre les porteurs des projets et les opposants sont d'autant plus marquées qu'elles s'inscrivent dans des «enjeux d'autoréférence d'une communauté à son territoire » (Plottu, 2015).

1. Sur les notions de représentations et perception, $c f$. Jodelet (1993).

\section{Matériel et méthode}

Tout d'abord, il faut préciser que les conflits étudiés ici ont la particularité d'avoir pris forme à l'occasion d'une Enquête publique (EP), à laquelle l'éleveur a obligation de soumettre son projet lorsque l'envergure de celui-ci dépasse le seuil défini par le Code de l'environnement ${ }^{2}$. Celui-ci précise que l'enquête prévoit «l'information, la participation et la prise en compte des intérêts des tiers »(Goutal et al., 2012). Pour une durée allant de deux semaines à deux mois, les habitants de la commune du projet ainsi que des communes limitrophes ou concernées par le plan d'épandage sont invités à consulter en mairie le dossier de l'étude d'impact du projet et à émettre un avis motivé sur celui-ci sur le registre d'enquête publique ou auprès du Commissaire enquêteur (CE) lors de ses permanences. À l'issue de cette phase d'enquête, le commissaire enquêteur rend un avis (favorable ou défavorable au projet) au préfet de région, et c'est ce dernier qui prendra seul la décision finale d'autorisation administrative du projet, suivant ou non l'avis du commissaire enquêteur. Il s'agit ainsi d'une démarche de « consultation » (voire de concertation) du public, si l'on suit l'échelle de participation définie par Arnstein (1969) qui distingue les différentes formes de participation selon le degré d'implication des citoyens, depuis la communication jusqu'à la négociation.

2. La réglementation sur les installations classées en élevage mise en œuvre à partir du $1^{\text {er }}$ janvier 2000 impose la réalisation d'une enquête publique pour tout projet concevant la présence sur un même site de plus 450 «porcs équivalents », soit 45 truies naisseur-engraisseur. La taille moyenne des élevages naisseurs engraisseurs bretons étant de 168 truies en 2000 et 190 en 2010 (source : Agreste Bretagne, 2012), la plupart des projets d'extension conduits en Bretagne au cours de la période d'enquête (2000-2012) ont nécessité le passage en enquête publique. 


\section{1. Échantillonnage des projets et des individus enquêtés}

L'échantillon des conflits comprend une diversité de projets (nature et dimension) et de contextes géographiques et sociaux. Les différents cas ont été identifiés grâce à des entretiens exploratoires menés en 2012 auprès de sept techniciens en charge de l'accompagnement des éleveurs lors de la constitution des projets et de la rédaction des dossiers d'étude d'impact.

Pour chacun des cas, une pré-enquête croisant les documents d'EP consultés en mairie ou en préfecture (résumé de l'étude d'impact, registre des plaintes, différents courriers envoyés au $\mathrm{CE}$, etc.) et des articles issus de la presse quotidienne régionale (recensés à l'aide du logiciel Europress) nous a permis d'identifier les principaux acteurs impliqués dans les conflits : opposants, défenseurs et témoins des projets. Parmi eux, nous avons sélectionné des individus concentrant des informations sur les conflits allant au-delà de leur histoire personnelle (personnes « leader » et/ou ayant suivi le projet en intégralité). Nous avons également privilégié des personnes résidant localement.

Suivant cette méthode, nous avons identifié 16 cas de conflits pour lesquels nous avons rencontré les porteurs de projet. Nous avons ensuite écarté six cas : pour trois d'entre eux l'opposition était limitée et/ou reposait sur des difficultés administratives plutôt que sociales, deux étaient portés par des éleveurs confrontés au moment de l'enquête à des difficultés personnelles et un projet était très atypique par sa dimension plus industrielle qu'agricole.

Les dix conflits retenus ont débuté entre 2001 et 2012. Il s'agit majoritairement de naisseurs engraisseurs ayant des projets d'extension visant à augmenter le nombre de truies et/ou atteindre une autonomie d'engraissement. L'échantillon inclut aussi un cas de délocalisation d'élevage pour s'éloigner d'un bourg et un cas de création de maternité collective (tableau 1). Au total, afin de rendre compte et d'analyser la complexité des situations et de valoriser une approche symétrique, nous avons rencontré 12 défenseurs de projets (dont 10 éleveurs-porteurs), 11 témoins, et 11 opposants.

\section{2. Élaboration des trames d'entretien et traitement des données}

L'analyse de la perception des projets par les différents acteurs repose sur la conduite d'entretiens semi-directifs élaborés en fonction de la position de la personne rencontrée. La trame d'entretien destinée aux opposants et témoins aborde (i) le récit de vie de la personne interrogée, (ii) le déroulement du conflit et (iii) la perception a posteriori du conflit. Celle mise en œuvre avec les éleveurs-porteurs intègre également (iv) la description du projet ayant rencontré une opposition et (v) l'avis sur d'autres projets contestés. Les entretiens ont été retranscrits intégralement et des extraits sont repris ici pour illustrer les résultats (en italique et entre guillemets).

Tableau 1. Caractéristiques des 10 projets retenus

\begin{tabular}{lcccccccccc}
\hline Exploitation & A & C & E & I & J & K & M & N & O & P \\
\hline Naisseur-engraisseur (nb truies)* & 280 & 160 & 500 & 450 & 450 & 85 & & 500 & 240 & 270 \\
\hline Naisseur (nb truies)* & & & & & & & 500 & & & \\
\hline Extension & $\mathrm{X}$ & & $\mathrm{X}$ & $\mathrm{x}$ & $\mathrm{x}$ & $\mathrm{x}$ & & $\mathrm{x}$ & $\mathrm{X}$ & $\mathrm{X}$ \\
\hline Création site porcin & & & & & & & $\mathrm{X}$ & & & \\
\hline Changement de site & & $\mathrm{X}$ & & & & & & & & \\
\hline
\end{tabular}

Note : * à l'issue du projet. 
Les éléments relatifs au déroulement des conflits et aux acteurs impliqués ont été rassemblés dans une grille de lecture croisant des informations sur les différents enquêtés: leur profil, leurs visions du contexte de la commune et de l'agriculture, leur perception du projet, du conflit et des autres acteurs concernés par le conflit, leurs arguments, actions et stratégies et leur prise de recul sur le conflit. La grille de lecture ainsi obtenue a ensuite été divisée pour obtenir d'une part une grille par population d'enquêtés (opposants, témoins, et défenseurs), et, d'autre part, une grille par cas de conflit comportant tous les individus enquêtés sur un même cas.

\section{Profils, représentations et connaissances des personnes enquêtées}

Les éleveurs porteurs de projets sont tous des hommes en âge d'être en activité. Six d'entre eux sont impliqués dans des structures d'ordre paraprofessionnel (syndicat, groupement de producteurs, chambre d'agriculture) et cinq ont des mandats politiques au sein d'un conseil municipal. Parmi ceux-ci figurent des personnalités connues pouvant incarner un modèle d'agriculture, un syndicat agricole ou encore un courant politique. À l'inverse, les opposants aux projets que nous avons rencontrés sont très majoritairement des retraités, hommes et femmes, ayant occupé des fonctions de cadres ou exercé des professions intermédiaires, n'ayant pas toujours habité dans leur milieu de vie actuel. Ils sont fortement impliqués dans une voire plusieurs associations (environnementales, culturelles, sportives...).

Les éleveurs considèrent souvent les opposants comme des personnes peu ou pas concernées par le contexte économique de leur profession et comme des « intégristes » de l'écologie. Ils distinguent néanmoins deux types d'opposants : ceux qui refusent le débat, et ceux avec qui le dialogue peut s'établir malgré des positions et arguments très différents : "Cette personne-là, elle a une étiquette politique écologiste. Elle est très agréable, très ouverte. Dans certaines associations, il y a des gens, tu ne peux rien leur dire, mais pas elle: tu peux discuter énormément avec elle. »

À l'inverse, chez les autres acteurs enquêtés, nous avons identifié quatre thématiques négatives fréquemment associées aux éleveurs porcins: (i) leur méfiance, interprétée soit comme une forme de timidité soit liée au fait d'avoir quelque chose à cacher, (ii) leur cupidité jugée plus spécifique de l'élevage porcin que des autres filières d'élevage, (iii) leur violence ou la dureté de leurs attitudes et propos ( $L e$ défaut qu'ils ont, c'est qu'ils seraient plus revendicatifs et manifestants que communicants et ouverts ») et (iv) leur malhonnêteté ("Il avait fait beaucoup de promesses qu'il n'a jamais tenues »). Pour les enquêtés ayant une vision négative de l'élevage et des éleveurs porcins, une cinquième caractéristique s'ajoute : (v) la dépendance des éleveurs à un système mis en place par d'autres acteurs de la filière.

Enfin, tous les acteurs impliqués dans les conflits ont capitalisé des connaissances sur l'élevage et l'agriculture. Celles-ci sont de source et de nature variées, et trois catégories sont à distinguer : (i) une connaissance « interne » pour les agriculteurs et les membres d'Organisations professionnelles agricoles (OPA), (ii) une connaissance par un lien personnel fort avec l'agriculture et (iii) une connaissance externe à partir de lectures, documentaires ou de rencontres avec des éleveurs. Le conflit est l'occasion pour ceux qui maîtrisent moins les contextes et référentiels des élevages porcins d'accroître leurs connaissances. C'est notamment le cas de certains opposants aux connaissances externes qui peuvent parfois «se forger des convictions », à 
partir des arguments d'autres opposants acteurs du milieu agricole.

\section{Attitudes initiales et registres d'argumentation}

\section{Un effet de surprise partagé}

Les éleveurs-porteurs apparaissent toujours convaincus du bien-fondé de leur projet et la plupart d'entre eux estiment ne pas devoir le justifier auprès de tiers. Ils le défendent par des arguments avant tout techniques et économiques (accéder à des économies d'échelles ou se conformer à de nouvelles réglementations) mais aussi sociaux (pouvoir travailler à plusieurs et ainsi améliorer leur qualité de vie). Immergés dans le référentiel technico-économique de leur profession, leurs projets leur paraissent tellement communs qu'ils ne comprennent pas qu'ils puissent engendrer une opposition: "C'est de l'épicerie et on en fait tout un pataquès », explique un éleveur. Ils soulignent aussi la conformité juridique du projet qui leur confère, selon eux, la légitimité suffisante pour se prémunir de tout risque de contestation.

C'est d'ailleurs l'absence de questionnement initial sur la perception du projet par des tiers qui conduit la majorité des éleveurs à ne pas juger utile de le présenter pour l'expliquer. Ce manque d'anticipation s'explique aussi par la référence à des projets antérieurs, au sein de la même exploitation ou de fermes voisines, n'ayant pas rencontré d'opposition. Cela fait également écho aux difficultés qu'éprouvent parfois les éleveurs-porteurs à présenter leurs activités, en raison de difficultés relationnelles individuelles, ou lorsque l'évolution de la population locale a conduit à une perte de lien social, ou encore parce que la mise en œuvre d'une communication compréhensible de tous, sans vocabulaire technique, leur paraît souvent ardue. Si bien que finalement, neuf des seize éleveurs rencontrés n'ont pas mis en œuvre de communication préalable pour expliquer le contenu et l'objectif de leur projet.

Sept d'entre eux ont toutefois présenté le projet aux conseils municipaux consultés et parfois aux riverains. Dans le cas de certains dossiers emblématiques, ils ont également contacté des opposants pressentis parmi les représentants syndicaux et/ou professionnels pour en expliquer les objectifs. Mais, la démarche de communication mise en œuvre par les éleveurs prend souvent la forme d'une tentative de réponse trop tardive ou trop défensive pour permettre d'instaurer un véritable dialogue avec les riverains. À l'inverse, les éleveurs ayant réussi à instaurer un dialogue, ainsi que les techniciens, soulignent l'importance de ce type de démarche. C'est le cas d'un éleveur confronté à un conflit lors d'un premier projet (pour lequel il n'avait pas communiqué) et ayant fait le choix lors d'un projet ultérieur, d'informer rapidement les riverains. Il considère aujourd'hui que : «Ça s'est très bien passé. Ils avaient beaucoup d'a priori. »

Dans la plupart des cas, qu'il y ait eu communication ou pas, les éleveurs sont surpris de la survenue d'une opposition ou du moins de son importance. Cette surprise s'appuie sur leur sentiment d'une part de porter un projet utile et juridiquement non contestable, et, d'autre part, d'être situé dans un contexte sans tensions : "Je pensais que tout allait bien dans le meilleur des mondes, mais ça n'est pas aussi net que ça. L'enquête publique a été révélatrice de tout ça. Je suis tombé des nues. » Les opposants soulignent également fréquemment la surprise ressentie à l'annonce du projet, notamment parce qu'ils n'ont pas toujours été informés directement par l'éleveur. Cela correspond aussi au sentiment d'assister à la dégradation d'un cadre de vie idéalisé : "On est tombé sur cette ruine qui nous a séduits. On s'est mis à la rénover. À chaque période de vacances, on venait y travailler pour pouvoir venir $y$ 
vivre à la retraite. [...] Et donc ce projet, un an à peine après qu'on se soit vraiment installé, nous tombe dessus.»

\section{Une incompréhension mutuelle des arguments des autres parties prenantes}

Selon les éleveurs-porteurs et leurs techniciens, la contestation s'appuie plus souvent sur la critique d'un modèle d'élevage jugé industriel et ses impacts sur l'environnement, que sur la contestation d'un aspect précis du projet. Selon les éleveurs, leur projet sert alors de bouc émissaire, ce qui fait dire à l'un d'entre eux : «En fait, c'est pas le projet qui les intéresse. C'est casser l'élevage industriel.» Les articles de la presse quotidienne régionale reprennent ces arguments des opposants : «Encore une fois, on n'est pas opposés aux cochons. C'est le modèle agricole tel qu'on veut nous l'imposer que nous contestons »(Ouest-France, 18/12/2008). Les opposants insistent sur l'effet précurseur que pourrait avoir ce projet : «Un des responsables disait 'Des [projets] comme ça, c'est un modèle! Y'en aura d'autres, et il faudra s'y faire!' Donc y'avait pour nous un projet d'envahissement, partout où il restait encore de la place.» Ces arguments généraux sont effectivement mis en avant par tous les opposants rencontrés mais certains avancent aussi des observations spécifiques aux projets. Néanmoins, ce résultat est certainement lié à notre choix d'enquêter des opposants au niveau d'implication important: cela se traduit très probablement par une meilleure connaissance des dossiers que celle d'observateurs venant uniquement signer le registre d'enquête publique.

\section{Dynamique des conflits}

\section{Motivations initiales et émergence du conflit}

Les conflits locaux doivent également être envisagés comme des controverses sociotechniques illustrant l'évolution récente des rapports entre science et société. Les porteurs de projets et leurs partenaires se positionnent en général comme des experts par opposition à des opposants qu'ils considèrent comme des profanes. Cependant, l'évolution des rapports entre science et société permet aux individus de mettre en avant d'autres formes de connaissance, tirées de leurs expériences directes, des « savoirs de plein air» (Callon et al., 2001) qui s'imposent dans les nouvelles arènes de débat dont font partie les enquêtes publiques. De ce fait, l'objet des conflits ne correspond plus seulement à l'usage partagé du territoire, soulevant la question des nuisances et de l'usage des terres, mais englobe également une controverse sociotechnique relative au modèle agricole et aux impacts environnementaux, économiques et sociaux des projets.

Cependant, tous les acteurs des conflits ne reconnaissent pas toujours ces deux objets. Les opposants insistent essentiellement sur ce caractère sociotechnique de la controverse, justifiant ainsi leur volonté de s'inscrire dans une action citoyenne et réfutant l'idée de la défense d'intérêts personnels. Les défenseurs des projets, quant à eux, mettent en avant le conflit d'usage. Ils refusent souvent le principe d'une controverse sociotechnique car ils estiment que les opposants n'ont pas de connaissances sur l'élevage porcin et qu'ils sont peu concernés par les réalités économiques (Grannec et al., 2014). Ils associent alors les revendications des opposants à la défense d'intérêts personnels et à une position nimbyste ${ }^{3}$.

En effet, certains mouvements d'opposition contre des projets de dimension modeste (dans deux cas) voire en rupture avec le modèle d'élevage contesté (dans le

3. NIMBY ou Nimby est l'acronyme de l'expression Not In My BackYard, qui signifie " pas dans ma cour ». Il est utilisé pour décrire une opposition locale par crainte de nuisances ou de préjudices personnels. 
cas d'un projet d'élevage biologique sur paille) sont assimilables à ces mouvements nimbystes; mais l'analyse croisée des discours conduit à relativiser son rôle. $\mathrm{Si}$ l'existence d'inquiétudes personnelles et de ressentiments d'origines variées constitue effectivement des motifs de mobilisation initiale, les mobilisations conduisent les riverains à élargir leur questionnement. La protestation initiale se transforme alors en une réelle controverse sociotechnique (Barbier, 2002) dépassant le seul intérêt des riverains, lesquels peuvent rejoindre des associations environnementales voire parfois se mobiliser contre d'autres projets non liés à leur cadre de vie et, par conséquent, ne relevant plus d'un effet Nimby. De plus, les mobilisations initiales contribuent également à réveiller des tensions multiples, parfois anciennes, liées à l'exploitation (nuisances engendrées par l'élevage, acquisition de terres...) ou non (rivalités politiques, professionnelles...). L'intégration de facteurs historiques et culturels et l'élargissement des revendications montrent ainsi que le seul effet Nimby ne peut suffire à expliquer l'évolution vers un conflit, comme d'autres avant nous l'ont bien montré (Barbier et Rémy, $2012)^{4}$.

\section{Mise en réseaux}

Malgré une diversité de motivations initiales, différents acteurs s'associent ponctuellement pour s'opposer à un projet. Le conflit prend alors naissance, se poursuit avec l'implication d'autres individus et aboutit à la mise en place d'un réseau

4. Il faut noter ici que certains travaux nuancent les propos des opposants selon qu'il s'agit de nouveaux venus ou de résidents déjà installés (newcomers and long terme residents, Smith et Kranich, 2000) : les premiers cibleraient plutôt des arguments relatifs à la préservation et à l'usage récréatif de l'espace, les seconds mettraient en avant l'utilisation des ressources et le développement économique. Toutefois, nous n'avons pu dégager de telles tendances dans notre étude. constituant une opposition collective, en réaction au sentiment de n'être représenté ni par les politiques ni par les scientifiques (Barbier et Rémy, 2012). Initiés par des acteurs touchés personnellement par un problème, ils peuvent être rejoints par de grandes organisations, régionales ou nationales. L'articulation entre le niveau local et l'échelle globale est facilitée, dans tous nos cas d'étude, par le fait que certains acteurs locaux sont également proches d'une organisation de plus grande ampleur ou la rejoignent à l'occasion du conflit. L'éleveur-porteur peut lui aussi constituer un réseau de défense, comportant également des acteurs locaux ou pas, aux profils relativement hétérogènes (agriculteurs aux visions différentes du métier, voisins, représentants d'OPA).

La présence d'agriculteurs dans les réseaux de soutien comme dans ceux d'opposition, impliqués personnellement ou via leurs organisations professionnelles ou syndicales, souligne que la survenue de conflits ne peut être résumée à une opposition entre monde agricole et non agricole. Cette mobilisation agricole joue d'ailleurs un rôle essentiel. S'il s'agit d'un positionnement contre le projet, elle permet aux autres opposants d'accroître leurs connaissances sur le sujet et de légitimer leur cause et leurs arguments. L'opposition agricole illustre parfois des rivalités personnelles, politiques, pour du foncier... Elle souligne aussi les divisions au sein du monde agricole, notamment entre les partisans d'un modèle agricole conventionnel « intensif » et les défenseurs d'une agriculture «plus familiale » voire « alternative ». Le projet devient alors le symbole de cette opposition dont l'ampleur et les enjeux dépassent les arguments situés de l'éleveur-porteur.

\section{Stratégies employées}

Malgré une diversité de situations, les opposants adoptent plus volontiers une stratégie de publicisation des conflits (Lemieux, 
2007). Cela correspond à leur volonté d'enrôler d'autres publics, notamment en réaction au sentiment de ne pas être suffisamment écoutés lors des procédures d'EP. Cette publicisation se fait via des réunions ouvertes, manifestations et pétitions. Les opposants peuvent également procéder par voie de recours juridique, par recherche de « failles » dans les dossiers. Ils considèrent que ces actions sont contraignantes et chronophages, mais nécessaires car constituant le seul moyen d'action efficace.

Les éleveurs estiment souvent que les opposants prennent plaisir à s'opposer, qu'ils le font par ennui. Ils tendent alors à ridiculiser leurs modalités d'action («Ils font le clown, à se déguiser en cochons devant la mairie, ça rime à rien »), ou à contester leur objectif («Ce qu'ils recherchent, c'est se faire entendre, se faire connaître [...], ils sèment le trouble, ils jouent sur la peur»). Quant à eux, ils adoptent généralement une stratégie de confinement de la controverse, notamment en ne répondant pas aux sollicitations des médias et associations, soit par prudence ou méfiance, soit par sentiment élitaire («Ils ne font même pas la différence entre une truie et un charcutier, il n'y a rien à discuter avec eux»). Cette logique de confinement peut être perçue par les opposants au mieux comme une impolitesse, au pire comme un signe de malhonnêteté.

\section{Analyse des conséquences et des déterminants des conflits}

\section{Conséquences sur les acteurs et les projets}

Pour les éleveurs-porteurs, le projet constitue un moment clé dans l'historique de leur exploitation, affectant non seulement leur vie professionnelle, mais également leur vie privée. Une opposition, même modérée, peut alors être anxiogène car il n'est pas possible de prévoir ni son ampleur ni ses conséquences. Ils jugent parfois que leur souffrance est sous-estimée par les techniciens des groupements : «Le groupement nous a peu soutenus. Il disait que c'est normal. Il ne trouvait pas notre cas important par rapport à d'autres projets. »

Le stress ressenti correspond tout d'abord à l'inquiétude de devoir abandonner le projet et aux conséquences sur la pérennité de l'exploitation. En effet, les conflits sont rarement sans impact sur le déroulement des dossiers : sur les conflits étudiés entre 2012 et 2014 seuls cinq d'entre eux ont abouti sans modification ni complication particulière. En revanche, en raison de l'opposition rencontrée, quatre éleveurs ont dû déposer un nouveau dossier et cinq n'ont pas obtenu l'autorisation d'exploiter ou l'ont vue annulée. Par ailleurs, les critiques interpellent l'éleveur en tant que représentant d'une profession et de ses pratiques (Bonnaud et Nicourt, 2006), comme cela transparaît dans les récits : «Moralement c'est très difficile d'être pris pour des menteurs, des pestiférés, des pollueurs. J'en ai encore la chair de poule. » De plus, le caractère public voire médiatique du conflit est perçu comme une atteinte à la vie privée et une attaque contre l'individu et est alors particulièrement mal vécu. En effet, les cadres de la vie privée et professionnelle étant souvent étroitement imbriqués, les critiques affectent également les proches et peuvent s'exprimer hors du cadre professionnel. Enfin, puisque le conflit est presque toujours initié par des acteurs locaux, potentiellement des voisins ou des relations personnelles, il est parfois vécu comme une trahison (Nicourt et Gasparo, 2005).

Comme pour l'ensemble des éleveursporteurs, les termes utilisés par la plupart des opposants ( $s e$ battre», «s'opposer ») soulignent un haut degré de conflictualité. Seuls deux opposants n'ont pas une vision conflictuelle de ce qui s'est passé : «On ne s'oppose pas, on donne un avis », résume l'un d'eux. Ils peuvent, à l'image 
des éleveurs, être affectés par le conflit, comme le décrit un témoin : «Ça a été tellement dur [...] ils ont été marqués quand même, les voisins, tous hein, vu comment ça s'est passé. »

Finalement, opposants et partisans des projets endossent chacun le statut de victime et la posture d'impuissance face à l'autre partie: les opposants mettent en avant le pouvoir des OPA et des lobbies porcins («on est en république cochonnière ») tandis que les éleveurs soulignent les compétences et les appuis juridiques des associations environnementales : «Je me sentais fort. Mais ça n'a pas pesé: les juges ce sont des citadins plus que des ruraux, voire des écolos convaincus.» Chacun est donc impliqué émotionnellement dans le conflit. Par ailleurs, plus les acteurs en opposition sont géographiquement proches, plus ils sont affectés. Quant aux acteurs plus éloignés, l'implication reste externe à leur sphère privée et relève du plus court terme, généralement la durée du conflit. C'est pourquoi, si tous les éleveurs-porteurs sont affectés, l'implication émotionnelle est plus variable pour les autres acteurs.

\section{Principaux déterminants}

L'ensemble des résultats permet d'affirmer qu'au-delà des motivations individuelles des acteurs impliqués, cinq traits dominants sont à l'origine des conflits.

i. Le contexte social emblématique : des territoires où la campagne est largement perçue comme «cadre de vie»: zones péri-urbaines ou littorales notamment. Dans ces cas, l'opposition est majoritairement partagée par les acteurs du niveau local et la revendication est forte. C'est le déterminant le plus fréquent.

ii. Le contexte environnemental emblématique (par exemple, les bassins algues vertes) conduit à étendre géographiquement le conflit et à impliquer d'autres populations et des acteurs emblématiques. Ce sont ainsi des conflits d'intensité très forte entre personnes qui ne se connaissent pas personnellement.

iii.La présence d'acteurs emblématiques (par exemple, des éleveurs ou des militants écologistes connus) dans le périmètre du projet. Ce déterminant semble présent dans la plupart de nos cas, dans une plus ou moins grande mesure. Dans ce cas, les conflits ont lieu entre des personnes se connaissant et ayant une histoire commune.

iv. La nature du projet impacte peu la perception du projet, hormis pour deux cas emblématiques en raison d'une dimension atypique ou d'un procédé novateur.

v. Les relations de voisinage et les comportements individuels des acteurs jouent un rôle d'autant plus important que la proximité (personnelle, géographique) est forte entre les protagonistes.

D'autres paramètres influent également sur l'apparition des conflits. En toile de fond, la densité porcine du territoire joue un rôle préventif : plus elle est forte, plus celle-ci semble être acceptée dans le territoire, et moins les conflits locaux sont intenses et étendus socialement. Néanmoins, lorsque cette forte densité porcine est associée à un contexte environnemental sensible, le conflit peut être intense et étendu géographiquement, par l'implication d'associations environnementales et des médias. Un autre déterminant est relatif à la communication. Si les éleveurs estiment suffisant d'avoir satisfait l'exigence réglementaire d'information du public via l'affichage et la publication dans la presse d'annonces, cette démarche est généralement inadaptée aux attentes des tiers car jugée par ces derniers trop impersonnelle et peu compréhensible, comme en témoigne une opposante après lecture de panneaux d'information: «Et alors, sauf si on connaît, on passe devant ces panneaux on ne sait pas ce que c'est. [...] C'est 
un langage abscons pour quelqu'un qui ne connaît pas : droits à produire, machin, truc. » D'autres éleveurs ayant communiqué en direction des élus et des voisins les plus proches pensent avoir suffisamment informé «les personnes concernées». Mais beaucoup de riverains ne font pas partie des personnes prévenues et prennent connaissance du projet seulement au moment de l'enquête publique, ce qui est souvent déjà trop tard pour établir un dialogue. L'incompréhension induit alors une inquiétude, et les riverains se tournent vers d'autres acteurs, parfois issus du monde agricole, pour se faire expliquer le projet.

\section{Préconisations en faveur de la concertation}

Afin que le conflit joue pleinement son rôle social, une démarche de sortie doit être mise en œuvre, instaurant un espace de débat constructif et permettant une évaluation ouverte du projet et son appropriation par l'ensemble des protagonistes. Nous proposons quelques éléments favorisant la prévention, la régulation ou la minimisation des conflits liés aux projets d'élevages : sans nier la vocation intégratrice du conflit, ses effets les plus néfastes sur les porteurs et les opposants pourraient être réduits.

\section{Passer d'une stratégie de communication à une démarche de concertation}

L'objectif affiché de l'enquête publique telle qu'elle est définie par la loi ${ }^{5}$ et comme cela est repris par le Code de l'environnement ( $c f$. supra) est bel et bien de concevoir une concertation. Néanmoins, et cela est d'autant plus vrai pour les cas étudiés

5. Notamment depuis la loi Bouchardeau de 1983, et plus encore depuis la réforme sur la démocratie de proximité de 2002 (Loi n 2002-276 du 27 février 2002 relative à la démocratie de proximité, $c f$. legifrance.fr). survenus avant 2002, la procédure intervient au stade final du processus de décision, alors même que l'éleveur a déjà obtenu le permis de construire, et ne permet pas d'apporter de modifications substantielles au projet sans la réalisation d'une seconde enquête. Cette limite importante des procédures de démocratie participative a déjà été soulignée (e.g., Blatrix, 2009) ${ }^{6}$ car elle conduit à une incompréhension et une insatisfaction mutuelle.

Les éleveurs estiment pour leur part avoir satisfait les obligations réglementaires. Face à l'obligation d'enquête publique, ils se demandent comment communiquer sur leur projet pour obtenir l'adhésion du public, tout en regrettant le manque de dialogue. Les élus rencontrés, quant à eux, perçoivent plutôt les enquêtes publiques comme relevant de la consultation. Les riverains souhaiteraient pour leur part qu'il y ait concertation voire négociation de manière à obtenir une décision acceptée par tous.

Par ailleurs, si chacun semble avoir pu exprimer son point de vue, soit de manière directe soit par des porte-parole, la qualité des échanges apparaît mitigée. Une discussion argumentée peut parfois avoir lieu - c'est d'ailleurs les cas des conflits les moins virulents. Mais bien souvent elle laisse place à des attaques d' ordre personnel et les rares moments de rencontre entre parties prenantes se soldent alors en agressions verbales. Il apparaît donc que, parce que les règles du jeu des enquêtes publiques sont mal comprises et/ou non acceptées par les deux camps et que les échanges entre parties prenantes sont de mauvaise qualité et ne permettent pas la compréhension des arguments ou des motivations de l'autre partie, les enquêtes

\footnotetext{
6. Notons à ce propos qu'une nouvelle réforme de l'enquête publique est en cours, notamment pour remédier à ce problème (décret de 2011 en cours d'expérimentation, qui donnera lieu à un bilan en 2017).
} 
publiques n'ont pas permis l'établissement d'un dialogue constructif.

\section{De l'intérêt de la médiation pour favoriser la concertation}

Comment alors passer d'une stratégie de communication à une démarche de collaboration? Malgré des divergences, par exemple sur les visions de l'agriculture, le fait de partager une expérience commune, des savoirs ou des représentations, un territoire peut servir de point de départ à une concertation. En revanche, il semble souhaitable d'envisager une médiation entre éleveurs et néoruraux, lorsque ces derniers n'ont pas d'expérience du milieu agricole ou qu'elle n'est pas reconnue par les représentants traditionnels de ce milieu.

Nos enquêtes laissent penser que deux réseaux sont en capacité de faire émerger de tels médiateurs : le conseil municipal de la commune concernée et le quartier ou village du projet. Les élus locaux peuvent jouer ce rôle de médiation à condition de porter un intérêt à l'agriculture et s'ils ne sont pas eux-mêmes engagés dans le conflit. Certains riverains «anonymes » peuvent aussi endosser ce rôle, notamment des autochtones ayant des connaissances agricoles ainsi qu'une expérience du territoire mais également d'autres contextes, et disposant de temps pour rencontrer les différentes parties : c'est la figure du voisin retraité, connu localement et qui a à cœur de maintenir ou restaurer une cohésion sociale. Or il apparaît que ce sont davantage les partenaires professionnels qui sont sollicités par l'éleveur-porteur pour l'accompagner dans la démarche de présentation du projet: des représentants du groupement ou d'autres OPA sont amenés, de manière plus ou moins explicite, à endosser ce rôle de médiateur qu'ils ne peuvent pourtant assurer. En effet, en tant que partie prenante dans le conflit ils ne possèdent pas la neutralité requise pour cette posture de médiation. De plus, leur intervention conforte le sentiment des opposants que les éleveurs sont dépendants, voire victimes, d'un système mis en place par d'autres acteurs de la filière.

\section{Procéder par étapes} et de manière adaptée à la situation

Malgré des actions engagées depuis plusieurs années par les éleveurs et leurs organismes de référence, la récurrence des conflits laisse penser que la recherche d'une meilleure concertation doit également être envisagée au sein même du monde agricole. Ainsi, un certain nombre de techniciens ont le sentiment que les contestations d'autorisations d'exploiter sont rares («Moins de 1\%; ça reste marginal », pense l'un d'entre eux) et que leurs conséquences sont négligeables («Toutes les EP laissent un peu de traces mais bon l'éleveur il passe vite là-dessus. L'essentiel, c'est qu'il puisse faire son bâtiment, 6 mois après il a oublié », affirme un autre). Cela souligne que la question de l'intérêt de démarches de concertation reste à débattre face au principe encore souvent communément admis que "pour vivre heureux, vivons cachés », et à la démarche contraire de publicisation du conflit par les opposants.

Face à cela, les éleveurs peuvent envisager différentes étapes. La première consiste à sortir de cette logique de confinement en ne refusant pas les sollicitations qui leur sont faites par les riverains ou les médias. Ensuite, ils peuvent appuyer le positionnement, en tant que médiateur, de certains acteurs locaux en informant de leur projet un entourage géographique ne se limitant ni aux voisins les plus proches ni aux seuls acteurs du milieu agricole, et cela suffisamment en amont. La réunion publique est couramment envisagée comme un dispositif de concertation mais, en raison de l'intervention d'acteurs autres que les éleveurs-porteurs ou que les initiateurs de l'opposition, elle génère souvent des 
débats trop virulents pour susciter un débat constructif sur la réalité du projet. De ce fait, la visite de l'élevage avec explication du projet nous semble plus adaptée. Elle permet, via l'information et le dialogue, d'instaurer entre parties prenantes un lien favorisant la compréhension mutuelle, comme un opposant en témoigne : «On a été vraiment très bien accueilli avec un souci d'apport technique pour qu'on comprenne comment ça fonctionnait, c'était une très bonne initiative, j'ai appris des choses. [...] Ils ne m'ont pas convaincue de l'intérêt de ce modèle-là sur le long terme, mais ça m'a apporté plus de tolérance. » Elle peut ainsi permettre de sortir de l'opposition entre confinement et publicisation, et du clivage entre les notions de controverse technique et de conflit d'usage.

\section{Enjeux et perspectives pour et par un dialogue renouvelé}

La prise en compte de l'avis de l'ensemble des acteurs du territoire permet une analyse compréhensive et symétrique des conflits. Elle souligne que les actions engagées sont liées aux expériences personnelles, à des connaissances et représentations de l'élevage porcin variées. Cristallisés par des enquêtes publiques dont l'intérêt et les règles du jeu sont encore mal compris ou mal perçus, les conflits locaux peuvent être considérés comme des symptômes de la transformation du mode de décision et d'action publique tout autant que comme des symptômes des évolutions de l'espace rural et des difficultés des éleveurs à s'y adapter. Ils sont aussi le fruit d'attitudes et d'événements passés soulevés par les éleveurs eux-mêmes : "Il ne faut pas non plus se dédouaner, on a fait des bêtises: le trop d'épandage, les truies illégales et aussi parfois le côté m'as-tu-vu : ça a créé une certaine animosité dans la population agricole. » La volonté récente d'intégration des attentes sociétales par la filière porcine, ou les changements de techniques et de pratiques des éleveurs, ne suffisent pas à prescrire ce constat.

Le travail mené ici permet toutefois de sortir d'une analyse du conflit en termes d'enjeux stratégiques ou liés aux potentiels de développement local pour orienter la lecture vers un troisième type d'enjeux, liés à l'expérience des individus, à la dimension symbolique, voire ontologique du conflit. C'est cette dimension qui est d'ailleurs définie par Plottu (2015) comme fondamentale pour faire émerger une solution négociée dans la gouvernance territoriale, car elle incarne «l'autoréférence d'une communauté à son territoire »; mais elle demeure encore trop souvent en retrait dans l'analyse des problématiques contemporaines en dépit de ses apports pour aborder la gouvernance (Plottu, 2015), la biodiversité (Micoud, 2005), les conflits environnementaux (Mormont, 2006) ou encore les biens publics (Boudes et Darrot, 2016).

La prise en compte de cette dimension est d'autant plus fondamentale qu'elle rejoint des propos déjà tenus par de nombreux auteurs sur la nécessité d'une démocratie participative ancrée, dans notre cas, à un développement rural et soutenue par un système institutionnel. Sans en passer par la transformation d'un modèle, comme le proposent par exemple Wiskerke et al. (2003) lorsqu'ils opposent modernisation et développement rural, il faut a minima prendre acte de cette nouvelle dynamique de revendication. Si la filière porcine fait preuve depuis plusieurs années d'une réelle volonté d'intégration des attentes sociétales, nos cas d'étude soulignent néanmoins un degré de concertation faible, si ce n'est inexistant, lors d'enquêtes publiques conflictuelles. En favorisant l'instauration d'un dialogue avec la société et en prenant en compte, de manière symétrique, la diversité des arguments et de leur nature, la mise en place de démarches de concertation pourrait permettre non seulement aux éleveurs mais aussi à l'ensemble des acteurs 
de la filière porcine de réaliser un progrès à la fois social et technique par et pour une meilleure compréhension, par les autres acteurs des territoires, de ses contraintes et des enjeux de son évolution.
Cette étude a été réalisée avec la contribution financière du Compte d'Affectation Spéciale pour le Développement Agricole et Rural et du Conseil Régional de Bretagne.

\section{RÉFÉRENCES BIBLIOGRAPHIQUES}

Agreste Bretagne. (2012). Résultats du recensement agricole. Les naisseurs-engraisseurs concentrent $80 \%$ du cheptel porcin. Décembre, $\mathrm{n}^{\circ} 2012-7,6 \mathrm{p}$.

Arnstein Sherry R. (1969). A ladder of citizen participation. JAIP, vol. $35, \mathrm{n}^{\circ} 4$, pp. 216-224.

Barbier R. (2002). Comment étendre la démocratie aux projets techniques? Actes de la $4^{e}$ Rencontre Ville Management, pp. 181-197.

Barbier R., Rémy E. (2012). Les controverses environnementales publiques. In Barbier R., Boudes P., Bozonnet J.-P., Candau J., Dobré M., Lewis N., Rudolf F. (dir.), Manuel de sociologie de l'environnement, PUL, pp. 187-201.

Blatrix C. (2009). La démocratie participative en représentation. Sociétés contemporaines, $\mathrm{n}^{\circ}$ 74, pp. 97-119.

Boudes Ph., Darrot C. (2016). Biens publics : construction économique et registres sociaux. Revue de la régulation, publication en cours.

Bonnaud L., Nicourt C. (2006). Les éleveurs de porcs face à leurs détracteurs en Dordogne et dans le Finistère. Études rurales, $\mathrm{n}^{\circ}$ 177, pp. 55-68.

Callon M., Lascoumes P., Barthe Y. (2001). Agir dans un monde incertain: essai sur la démocratie technique. Paris, Le Seuil, $358 \mathrm{p}$.

Danel J.-B., $\quad$ Fouillade P., Guillet M., Travers J.-M. (2012). Quel avenir pour la filière porcine française? Rapport d'étude CGAAER, ${ }^{\circ} 11050,77 \mathrm{p}$.

Darly S. (2012). La reterritorialisation de l'agriculture, effet collatéral des conflits d'usages. Le cas francilien. Économie rurale, $\mathrm{n}^{\circ} 332$, pp. 31-46.
Delanoue E., Roguet C., Selmi A. (2014). Contestation sociale de l'élevage porcin en France : regards croisés de professionnels de la filière et d'associations. Journées de la recherche porcine, $\mathrm{n}^{\circ} 46$, pp. 235-240.

Duhaime J. (2001). Le conflit comme socialisation selon G. Simmel. Communication au Congrès de l'ACFAS, $19 \mathrm{p}$.

Dziedzicki J.-M. (2003). La gestion des conflits d'aménagement entre participation du public et médiation. Annuaire des collectivités locales, vol. 23, $\mathrm{n}^{\circ}$ 1, pp. 635-646.

Goutal Y., Peynet P., Peyronne A. (2012). Droit des enquêtes publiques. Lamy, Axe Droit, $278 \mathrm{p}$.

Grannec M.-L., Ramonet Y., Selmi A. (2014). Les projets d'élevages porcins en Bretagne. Perception des conflits et de leurs enjeux par les acteurs de la filière. Journées de la recherche porcine, $\mathrm{n}^{\circ} 46$, pp. 241-246.

Jodelet D. (1993). Les représentations sociales. Paris, PUF, $424 \mathrm{p}$.

Légifrance 2002, loi n 2002-276 du 27 février 2002, en ligne sur Légifrance.fr

Lemieux C. (2007). À quoi sert l'analyse des controverses? Mil neuf cent, $\mathrm{n}^{\circ} 25$, pp. 191-212.

Mathieu N. (1998). La notion de rural et les rapports ville/campagne en France: les années quatre-vingt-dix. Économie rurale, $\mathrm{n}^{\circ} 247$, pp. 11-20.

Micoud A. (2005). La biodiversité est-elle encore naturelle ? Écologie et Politique, $\mathrm{n}^{\circ} 30$, pp. 17-25.

Mormont M. (2006). Conflit et territorialisation. Géographie, Économie et Société, vol. 8, n³, pp. 299-318.

Nicourt C., Girault J.-M., Bourliaud J. (2000). Les odeurs d'élevages: textes, conflits et 
RECHERCHE

négociations locales, Économie rurale, Smith M., Kranich R. (2000). Culture clash $\mathrm{n}^{\circ} 260$, pp. $79-89$.

Nicourt C., Gasparo S. (2005). Critique du travail et fragilisation de l'identité de l'éleveur. Journées de la recherche porcine, $\mathrm{n}^{\circ} 37$, pp. 391-396.

Park R. E. [1926] (1979). La communauté urbaine : un modèle spatial et un ordre moral. In Grafmeyer Y., Joseph I., L'École de Chicago, Paris, Champ urbain.

Perrier-Cornet P. (2002). Repenser les campagnes. L'Aube, La Tour d'Aigues, $279 \mathrm{p}$.

Plottu B. (2015). Conflit d'usage du territoire : de l'identification des enjeux à l'émergence d'une solution négociée. Économie rurale, $n^{\circ} 348$, pp. 23-41.

Simard L., Lepage L., Fourniau J.-M., Gariépy M., Gauhtier M. (dir.) (2006). Le débat public en apprentissage: aménagement et environnement. Paris, L'Harmattan, $318 \mathrm{p}$.

Simmel G. [1908 (1999)] Sociologie : études sur les formes de la socialisation. Paris, PUF, $780 \mathrm{p}$. revisited: newcomer and longer-term residents' attitudes toward land use, development, and environmental issues in rural communities in the Rocky Mountain West. Rural Sociology, vol. 65, n 3, pp. 396-421.

Torre A., Aznar O., Bonion M., Caron A., Chia E., Galman M., Guérin M., Jeannneaux Ph., Kirat Th., Lefranc Ch., Melot R., Paoli J.C., Salazar M.I., Thinon P. (2006). Conflits et tensions autour des usages de l'espace dans les territoires ruraux et périurbains : le cas de six zones géographiques françaises. Revue d'économie régionale \& urbaine, $\mathrm{n}^{\circ}$ 3, pp. 415-453.

Thuderoz, C. (2014). Sociologie du conflit en entreprise. Rennes, PUR, 120 p.

Wiskerke J.S.C., Bock B.B., Stuiver M., Renting H. (2003). Environmental cooperatives as a new mode of rural governance. NJAS - Wageningen Journal of Life Sciences, vol. 51, n 1-2, pp. 9-25. 
